Pierre-Yves BEAUREPAIRE, Dominique TAURISSON (dir.), Les Ego-documents à l'heure de l'électronique. Nouvelles approches des espaces et réseaux relationnels

\title{
Raymonde Monnier
}

\section{(2) OpenEdition \\ 1 Journals \\ Édition électronique \\ URL : https://journals.openedition.org/ahrf/10362 \\ DOI : 10.4000/ahrf.10362 \\ ISSN : 1952-403X \\ Éditeur : \\ Armand Colin, Société des études robespierristes}

\section{Édition imprimée}

Date de publication : 15 mars 2006

Pagination : 244-246

ISSN : 0003-4436

\section{Référence électronique}

Raymonde Monnier, «Pierre-Yves beaurePAIRE, Dominique taurisson (dir.), Les Ego-documents à l'heure de l'électronique. Nouvelles approches des espaces et réseaux relationnels », Annales historiques de la Révolution française [En ligne], 343 | janvier-mars 2006, mis en ligne le 17 novembre 2008, consulté le 24 avril 2022. URL : http://journals.openedition.org/ahrf/10362 ; DOI : https://doi.org/10.4000/ahrf. 10362

Ce document a été généré automatiquement le 24 avril 2022.

Tous droits réservés 


\title{
Pierre-Yves BEAUREPAIRE, Dominique TAURISSON (dir.), Les Ego-documents à l'heure de l'électronique. Nouvelles approches des espaces et réseaux relationnels
}

\author{
Raymonde Monnier
}

\section{RÉFÉRENCE}

Pierre-Yves Beaurepaire, Dominique Taurisson (dir.), Les Ego-documents à l'heure de l'électronique. Nouvelles approches des espaces et réseaux relationnels, Montpellier, Publications de Montpellier III, 2003, 553 p., ISBN 2-84269-594-1, $30 €$.

1 Malgré son titre, ce livre rassemble des études qui pour la plupart témoignent moins d'une connaissance des nouvelles techniques d'analyse des textes et des traitements liés à la numérisation des corpus, qu'un intérêt commun pour l'histoire des espaces et réseaux relationnels dans l'Europe des Lumières, qu'ils soient confessionnels, diplomatiques, géographiques, professionnels ou familiaux. Le colloque international de Montpellier en 2002 qui est à l'origine de la publication s'inscrivait d'ailleurs dans une série de rencontres, initiées par un Réseau scientifique pour l'étude de la communication dans l'Europe moderne, notamment celle des correspondances et autres écrits du for privé. L'ensemble, dû à une trentaine d'auteurs, rassemble des études d'un intérêt inégal selon le sujet traité, et les méthodes mises en œuvre; cependant, quelques contributions sont particulièrement riches et permettent de prendre conscience de l'importance du champ de recherche, des avancées méthodologiques déjà réalisées et de dégager quelques pistes prometteuses quant à 
l'analyse de grands corpus d'ego-documents dans la perspective de la configuration de relations étendues et complexes.

La richesse du volume tient aussi à la diversité des approches, qui entraîne l'éventail des sources exploitées, pour ces histoires du réseau et de ses usages, qui croisent histoires de familles, histoires de carrières administratives ou commerciales, histoires socio-politiques. Sans doute, l'étude de la collection de cartes et du réseau de correspondants d'un grand commis de l'État (Coquebert de Monbret) permettrait-elle de comprendre la manière dont s'est en partie constituée la géographie, à la croisée de la méthode scientifique et du savoir administratif (I. Laboulais-Lesage). À travers sa Correspondance, le grand écrivain (Rousseau) ouvre à d'autres phénomènes de réseau et à la construction d'un système où l'œuvre se confond avec la destinée de l'écrivain (A.$\mathrm{F}$. Grenon et $\mathrm{F}$. Salaün). Les actes notariés et paroissiaux s'avèrent des plus pertinents pour l'approche ethnologique de la construction sociale, à travers les réseaux de parenté et l'esprit de "Maison» lié à la possession de terres et d'esclaves, dans le système du lignage qui préside au développement de l'île Bourbon (La Réunion), à l'ère du café et de la plantation esclavagiste (S. Tourreau). Dans un lignage construit à partir des réseaux de la Compagnie des Indes et étendu aux différents comptoirs français de l'Océan Indien, les liens symboliques avec les familles d'origine - banquiers et négociants protestants du Languedoc - ont tendance à se distendre au profit de la «Maison » et d'une identité créole, au sein de la vie politique et de la sociabilité locale, fondée sur l'appartenance au temple maçonnique, l'amitié, la confiance et l'échange de services.

3 Au-delà du cercle de famille, la notion d'entourage mise en valeur par les démographes, permet une analyse sociologique à partir de la topologie du réseau, « du petit monde » occasionnel ou permanent d'un auteur de livre de raison (S. Mouysset). L'anthropologie et la sociologie ont contribué à mettre en avant un certain nombre de notions analytiques pour dépasser l'usage métaphorique du concept de réseau et caractériser les liens (forts ou faibles) et les positions relatives des personnes qui participent de réseaux ego-centrés, en termes de configuration et de structuration des espaces sociaux (M. Gribaudi dir., Espaces, temporalités, stratifications. Exercices sur les réseaux sociaux, Paris, EHESS, 1998). On mesure ce que les notions sociologiques de capital social, de liens, d'action et de ressources peuvent apporter à l'étude de la stratégie des réseaux religieux en exil, qu'il s'agisse de la diaspora jacobite en France et en Italie (C. Prunier), ou des huguenots du Refuge (C. Berkvens-Stevelinck). L'identité de la communauté et sa survie même dépendaient de la stratégie consciente ou inconsciente de ses membres et des réseaux qu'ils avaient su mettre en place. L'étude de cas présentée par F. DeroyPineau et P. Bernard montre l'apport d'un outil anthropologique à l'analyse de la trajectoire d'une "étoile sociométrique » : la stratégie relationnelle d'une religieuse que rien ne semblait prédestiner à devenir la fondatrice des Ursulines du Canada (Sœur Marie de l'Incarnation), répond de sa part à un processus sociométrique parfaitement construit, secondé par une intelligence et un charisme hors du commun.

Dans la catégorie des ego-documents, les correspondances occupent une place de choix. Elles concernent plusieurs études du volume, traitées souvent de façon très classique. Le contenu d'une correspondance privée bordelaise fournit une approche de la culture matérielle autour des thèmes des plaisirs et des jours, qui gagnerait à être moins étroitement descriptive. La notion de réseau n'est-elle pas à la fois nécessaire et insuffisante pour appréhender ces textes, à la croisée de l'individuel et du social ? En 
prolongement de ses travaux sur les correspondances familiales, C. Dauphin pose le problème du traitement systématique de ce genre de corpus, émietté et lacunaire, dont l'architecture dépend de la valeur affective de certaines lettres, des événements qui touchent les proches, enfin des conditions et des gestes familiaux de conservation, euxmêmes liés à diverses relations de travail et d'intérêts, autour du socle naturel de la parenté et des figures qui valorisent le patrimoine commun. Comment configurer la nébuleuse des correspondants, déterminer les significations du jeu complexe des citations dans l'espace épistolaire, au sein des différents réseaux de relations, qu'ils relèvent de la parenté, du quotidien, de la sociabilité, de la profession ou de l'existentiel? L'expérience sur un échantillon restreint permet d'énoncer des hypothèses de travail. Un outil aussi perfectionné soit-il, peut-il restituer l'image, le graphe lisible du réseau d'échange, pour apprécier le rôle et les fonctions d'une configuration toujours changeante, soumise au jeu et à la plus ou moins grande liberté de ses acteurs, à la charnière de l'espace intime et de l'environnement social qui génère les pratiques d'écriture?

5 Que peut apporter le traitement informatique sur le réseau de relations et d'échanges et le contenu des correspondances? L'apport et les limites de la lexicologie quantitative à partir des divers logiciels d'analyse des textes (Lexico, Hyperbase, Wordmapper, Weblex) sont connus. Ces outils permettent une approche sans a-priori de gros corpus, mais nécessitent un traitement préparatoire spécifique assez lourd et un découpage chronologique pertinent pour dégager des facteurs d'évolution. L'analyse de la correspondance d'un curé béarnais, ancien secrétaire du cardinal Dubois, le montre bien (V. Castagnet). L'appauvrissement thématique et lexical qui se dessine en un peu plus de dix ans ne prend vraiment de sens que rapporté à d'autres éléments du contexte qui relèvent de la biographie historique. L'essai méthodologique de $\mathrm{Z}$. Moutoukias, sur le réseau ego-centré d'un grand personnage de l'oligarchie coloniale espagnole, démontre de façon convaincante à quel prix les résultats de la recherche peuvent compenser le poids de l'investissement initial qu'elle demande. Sa méthode analytique se donne les moyens de combiner, à l'aide d'une typologie élaborée, l'analyse de la représentation graphique complexe des points et des liens du réseau Belgrano avec le récit historique. En reconfigurant l'espace social et physique des liens d'un réseau ego-centré, les graphes font apparaitre le rôle important de certains relais liés à la dynamique du crédit. Ils montrent comment certains acteurs se trouvent au croisement d'une multiplicité de ressources spécifiques qui constituent un vrai capital.

Plus que des recherches abouties, les communications présentent des initiatives prometteuses et des projets en cours de réalisation par des bibliothèques ou des équipes universitaires, pour mettre en commun des ressources documentaires dispersées, inventorier sur la toile correspondances et livres de raison, constituer des bases de données qui donnent accès à la culture de l'Europe moderne. Citons, entre autres, le projet présenté par M. Bertrand sur les élites politiques et administratives de l'empire espagnol, ou encore dans le domaine de la bibliographie, la base de données Ipséité, projet d'inventaire raisonné des journaux et carnets personnels en langue française. Comme le remarque J. Boutier dans la postface, la saisie informatique des correspondances contribue a réhabiliter en l'enrichissant, une des activités les plus anciennes de l'historien, celle de l'édition critique des sources. La base de données ainsi constituée est immédiatement utilisable avec les outils pour la visualiser : c'est le cas de la base de données "Pierre Bayle", élaborée par A. McKenna et A. Leroux avec le logiciel Arcane, pour l'édition à la Voltaire Foundation de la Correspondance de Pierre 
Bayle. Autant de projets qui ont pour vocation de fédérer les initiatives, proposer des hypothèses de travail et favoriser l'échange en réseau entre chercheurs passionnés d'ego-documents. 